Pesq. Vet. Bras. 35(8):701-708, agosto 2015 DOI: $10.1590 / \mathrm{S} 0100-736 \mathrm{X} 2015000800001$

\title{
Infecção por Corynebacterium pseudotuberculosis em equinos: aspectos microbiológicos, clínicos e preventivos ${ }^{1}$
}

\author{
Maria T. Guedes², Bianca C. Souza른 Thiago J. Sousa ${ }^{3}$, Dan Loureiro², Lilia F. Moura- \\ Costa ${ }^{4}$, Vasco Azevedo ${ }^{3}$, Roberto Meyer ${ }^{2}$ e Ricardo W. Portela ${ }^{2 *}$
}

\begin{abstract}
Guedes M.T., Souza B.C., Sousa T.J., Loureiro D., Moura-Costa L.F., Azevedo V., Meyer R. \& Portela R.W. 2015. [Corynebacterium pseudotuberculosis infection in horses: clinical, microbiological and prevention aspects.] Infecção por Corynebacterium pseudotuberculosis em equinos: aspectos microbiológicos, clínicos e preventivos. Pesquisa Veterinária Brasileira 35(8):701-708. Laboratório de Imunologia e Biologia Molecular, Instituto de Ciências da Saúde, Universidade Federal da Bahia, Av. Reitor Miguel Calmon s/n, Vale do Canela, Salvador, BA 40110-100, Brazil. E-mail: rwportela@gmail.com

Corynebacterium pseudotuberculosis is the etiologic agent of caseous lymphadenitis in sheep and goats, an infectious disease that is responsible for significant economic losses in small ruminants breeding units worldwide. This Gram-positive bacterium can infect horses, causing symptomatic disease to systemic infections, which can lead to animals' death. Specifically in Brazil, there are no scientific records of equine infections, but it is believed that, due to the maintenance of infected small ruminants in close association to horses in many properties, the infection of horses may be a reality. The present work had the objective to present information on the bacteria C. pseudotuberculosis, on the epidemiological and clinical aspects of the infection in horses, as well as information about breeding procedures that can be adopted in order to prevent the infection.
\end{abstract}

INDEX THERMS: Corynebacterium pseudotuberculosis, horses, ulcerative lynphangitis, caseous lymphadenitis.

RESUMO.- Corynebacterium pseudotuberculosis é o agente causador da linfadenite caseosa em caprinos e ovinos, sendo responsável por significativas perdas econômicas na ovinocaprinocultura mundialmente. Esta bactéria Gram- positiva também infecta equinos, causando desde quadros assintomáticos até infecções sistêmicas, podendo levar o animal a óbito. Especificamente no Brasil, não foram relatados casos de infecção em equinos, mas acredita-se que, devido à convivência de pequenos ruminantes infectados

\footnotetext{
${ }^{1}$ Received on October 16, 2014.

Accepted for publication on June 29, 2015

${ }^{2}$ Laboratório de Imunologia e Biologia Molecular, Instituto de Ciências da Saúde, Universidade Federal da Bahia (UFBA), Av. Reitor Miguel Calmon s/n, Vale do Canela, Salvador, BA 40110-100, Brazil. * Corresponding author: rwportela@gmail.com

${ }^{3}$ Laboratório de Genética Celular e Molecular, Instituto de Ciências Biológicas, Universidade Federal de Minas Gerais (UFMG), Av. Presidente Antônio Carlos 6627, Belo Horizonte, MG 31270-901, Brazil.

${ }^{4}$ Laboratório de Microbiologia, Instituto de Ciências da Saúde, UFBA, Av. Reitor Miguel Calmon s/n, Vale do Canela, Salvador, BA 40110-100.
}

com equinos em diversas propriedades rurais, seja natural que ocorra a infecção desses animais. A presente revisão tem como objetivo fornecer informações sobre a bactéria C. pseudotuberculosis, sobre os aspectos epidemiológicos e clínicos da infecção em equídeos, bem como sobre técnicas de manejo para sua prevenção.

TERMOS DE INDEXAÇÃO: Corynebacterium pseudotuberculosis, equinos, linfangite ulcerativa, linfadenite caseosa.

\section{INTRODUÇÃo}

O gênero Corynebacterium pseudotuberculosis (do grego korune $=$ forma de clava e do latim = bacterium) causa doença de caráter crônico e contagioso em equinos, cuja forma clássica é conhecida por linfangite ulcerativa (LU) ou abscesso de peito, ou ainda como febre de pombo. Essa patologia foi primeiramente relatada por Hall \& Fisher (1915), que a descreveram como sendo caracterizada por abscessos intramusculares profundos, localizados no músculo peitoral, ombros e parede abdominal inferior (Doherr 
et al., 1998). Atualmente, vem-se estabelecendo um consenso comum no reconhecimento de três formas clínicas distintas da infecção em equinos: abscessos superficiais externos, abscessos internos ou forma visceral, e a linfangite ulcerativa propriamente dita (Hall et al. 2001, Pratt et al. 2005, Spier \& Whitcomb 2007).

Em 1888, Edmond Nocard, veterinário francês, descreveu pela primeira vez bactérias pleomórficas obtidas de material purulento oriundo de um caso de linfangite bovina. Três anos depois, em 1891, Hugo Von Preïsz identificou bactérias semelhantes às que Nocard havia relatado, numa cultura de abscesso renal de ovelha. Em homenagem aos dois pesquisadores, o microrganismo recebeu a designação de Bacilo Preïsz-Nocard. Em 1896, Lehmann \& Neumann, observando os tubérculos causados por esse bacilo, e verificando que não ocorria a formação de tubérculos 'verdadeiros' conforme os da tuberculose, renomearam-no Bacillus pseudotuberculosis. Em 1923, o microrganismo foi agrupado no gênero Corynebacterium, passando a ser chamado Corynebacterium ovis e, em 1948, recebeu a designação conforme conhecida hoje, Corynebacterium pseudotuberculosis. A primeira vez que C. pseudotuberculosis foi isolado e caracterizado no Brasil foi em 1973 por Moura-Costa, a partir de material de lesões em um caprino no interior da Bahia (Moura-Costa 2002, Dorella et al. 2006).

Inicialmente, a subdivisão da espécie $C$. pseudotuberculosis em dois biovares, o biovar ovis e o equi foi proposta por Biberstein et al. (1971). Posteriormente Songer et al. (1990) adotaram a mesma nomenclatura após análises do DNA cromossômico. Portanto, atualmente considera-se que a espécie $C$. pseudotuberculosis se subdivide nesses dois biovares: o biovar ovis ( $C p$ bv ovis) e o equi ( $C p$ bv equi) (McNamara et al. 1995, Silva et al. 2011). Soares et al. (2013a) demonstraram que os dois biovares, apesar de terem genótipos distintos, possuem a mesma origem evolucionária.

$O$ patógeno $C p$ bv ovis é responsável por infecção de ovinos e caprinos, apresenta resultado negativo na prova bioquímica do nitrato, é resistente à estreptomicina e $C p$ bv equi se caracteriza por ser nitrato positivo e sensível à estreptomicina (Sutherland et al. 1996, Costa et al. 1998). A interação imunológica microrganismo-hospedeiro de $C p$ bv ovis tem sido fartamente estudada e descrita; por outro lado, pouco se sabe sobre esta interação para $C p$ bv equi.

\section{CARACTERÍSTICAS MORFOLÓGICAS E BIOQUÍMICAS DE Corynebacterium pseudotuberculosis}

As bactérias pertencentes ao gênero Corynebacterium apresentam temperatura e $\mathrm{pH}$ de crescimento ótimos a $37^{\circ} \mathrm{C}$ e 7,0 , respectivamente, crescendo bem em ambiente com $5 \%$ de $\mathrm{CO}_{2}$ são Gram-positivas; medem em torno de 0,5 a $0,6 \mu \mathrm{m}$ por 1 a $3 \mu \mathrm{m}$, apresentando-se também sob forma de cocos ou filamentos. São anaeróbios facultativos, catalase e urease positivos, fermentam glicose e ribose, sem produção de gás, e cerca de $80 \%$ dos isolados fermentam a maltose, mas não fermentam lactose, manose e sacarose. Não produz esporos e são imóveis, possuindo pili, e são capazes de produzir biofilme (Moura-Costa 2002, Abreu et al. 2008a, Proft \& Baker 2009). Possuem a característica de serem intracelulares facultativas (Baird \& Fontaine 2007), não hidrolisando gelatina e nem digerindo a caseína (Songer et al. 1990). Apresentam parede celular com alta concentração de lipídios (Abreu et al. 2008a), particularmente o ácido corinomicólico (Spier \& Whitcomb 2007).

Crescem bem em ágar acrescido de $5 \%$ de sangue ovino, caprino, bovino ou equino, entre 24 e 72 horas de incubação, formando colônias que variam de branco-acinzentado a amarelado com aspecto opaco, e circundados por halo de $\beta$-hemólise (Pugh 2004). As colônias apresentam-se secas e não aderidas ao meio (Ribeiro et al. 2011) e não são álcool-ácido resistentes como as micobactérias (Motta et al. 2010).

\section{FATORES DE VIRULÊNCIA}

Os fatores de virulência do gênero Corynebacterium tem papel fundamental na adesão, invasão, colonização, propagação e na evasão do sistema imune do hospedeiro (Schumann, 2007). A camada lipídica complexa, presente na estrutura da parede celular pertencente ao grupo CMNR, grupo o qual envolve os gêneros Corynebacterium, Мycobacterium, Nocardia e Rhodococcus, já teve seu papel na virulência reconhecido, como por exemplo, conseguir se multiplicar dentro de macrófagos (Motta et al. 2010).

As fosfolipases formam um grupo heterogêneo de enzimas cuja atividade comum é a capacidade de hidrolisar uma ou mais ligações éster nos glicerol fosfolipídios (Spier et al. 2004, Soares et al. 2013b), e a PLD é uma exotoxina hemolítica capaz de promover hidrólise e degradação da esfingomielina em N-acetilesfingosil (Carne \& Onon 1978), aumentando localmente a permeabilidade vascular, o que contribui para a disseminação do patógeno no interior do hospedeiro (Hodgson et al. 1992, McNamara et al. 1994, Williamson 2001).

A PLD de $C p$ bv equi, assim como de $C p$ bv ovis, tem estrutura química semelhante à PLD produzida pela aranha marrom (Loxosceles $\mathrm{sp}$ ) e desenvolve patogênese similar, como a capacidade de aumentar a permeabilidade vascular, o que explica a presença de dor e edema no local da infecção (Spier \& Whitcomb 2007), e aumenta a capacidade de persistência e disseminação dessas bactérias dentro do hospedeiro (Cuevas \& Songer 1993), já que aquelas que apresentam mutagênese no gene $r p o B$ são PLD- e não desenvolvem a mesma virulência que as $\mathrm{PLD}^{+}$(Hodgson et al. 1992, McNamara et al. 1994, Spier et al. 2004).

Outro importante fator de virulência é a capacidade de produção de biofilme que o gênero Corynebacterium apresenta. Essa estrutura é um agregado de microcolônias envoltas por uma matriz de polissacarídeos, formando comunidades organizadas que permitem a comunicação entre si, aderindo a qualquer superfície biológica, o quê poderia explicar parte da resistência à ação dos antimicrobianos e biocidas (O'Toole et al. 2000, Olson et al. 2002, Hall-Stoodley et al. 2004, Clutterbuck et al. 2007). Em um estudo mais recente, 398 amostras de C. pseudotuberculosis foram testadas para verificação da formação de biofilme, e $25 \%$ delas demonstraram alta ou moderada capacidade de produção de biofilme; de forma interessante, foi verificado que o iodo diminuiu a formação de biofilme em 13 isolados e a amônia 
quaternária em 11 isolados (Sá et al. 2013). Estudos genômicos realizados com a cepa 258 de $C p$.bv equi estão revelando, por exemplo, detalhes dos processos biológicos de resposta a fatores de estresse, como $\mathrm{pH}$ ácido, tratamento térmico e concentração osmótica, os quais têm influência direta na formação ou não de biofilme (Soares et al. 2013b).

Os genes de Corynebacterium pseudotuberculosis mais estudados são aqueles vinculados à virulência, como é o caso do gene $r p o B$, cuja análise filogenética demonstra estar presente em todas as espécies do gênero Corynebacterium até agora estudadas, e sua expressão implica na produção da exotoxina PLD (Khamis et al. 2004, Soares et al. 2013b). Genes como: $A r o B$ e AroQ, que expressam enzimas envolvidas no processo de biossíntese de compostos nutritivos para a bactéria como fenilalanina, tirosina e triptofano; $f a g A, f a g B, f a g C$ e $f a g D$, envolvidos na captação de ferro do ambiente (D'Afonseca et al. 2008) expressam fatores de virulência de importância para a sobrevivência do microrganismo.

\section{EPIDEMIOLOGIA}

$C p$ bv equi é capaz de sobreviver em diferentes tipos de substrato e sob vasta gama de condições ambientais (Lazzari et al. 1997, Spier et al. 2012). Sobrevive por até dois meses em feno e aparas de madeira, e mais de oito meses no solo, especialmente naqueles ricos em ferro (Braverman et al. 1999, Spier \& Whitcomb 2007).

0 gênero Corynebacterium tem ampla distribuição mundial (Pratt et al. 2005) e alta adaptação evolutiva. A ocorrência deste gênero não está vinculada a uma localização geográfica em particular, nem mesmo a um número limitado de propriedades em uma determinada área (Woolcock et al. 1979). Já foi isolada desde animais de produção a ambientes e seres que vivem em situações extremas, como areia de deserto (Zhou et al. 2012), em solo salino (Chen et al. 2004), em focas (Bauwens et al. 1987, Collins et al. 2004), de esfregaço de queijo curado (Brennan et al. 2001), de corais marinhos (Ben-Dov et al. 2009), de sedimentos costeiros (Du et al. 2010) e até de combustível de origem microbiana (Wu et al. 2011).

A via de entrada no organismo do hospedeiro ocorre através de feridas na pele, abrasões, lesões por picada de insetos, membranas mucosas, por contaminação iatrogênica e também por via sanguínea (Hall et al. 2001, Spier \& Whitcomb 2007). Os casos de doença clínica, em pequenos ruminantes, podem ocorrer na forma de casos pontuais (Motta et al. 2010), ou sob a forma de surtos (Smith \& Robinson 1981, Pratt et al. 2005). Nos Estados Unidos, sua incidência é sazonal, sendo mais frequente nos meses secos (Doherr et al. 1998, Pethick et al. 2012, Spier et al. 2012).

Os nódulos linfáticos abertos, oriundos do desenvolvimento da infecção drenam material purulento, contaminando o solo, a água (Yeruham et al. 1996), as fezes, e fômites; objetos com superfície composta de aço, plástico ou madeira utilizados em animais infectados são considerados importantes fontes de contágio, bem como aparas de madeira, feno, palha, aos quais os equinos doentes tem acesso (Augustine \& Renshaw 1986). O solo tem sido re- latado na literatura como sendo o principal reservatório mantenedor da bactéria no ambiente (Robinson 1982, Spier \& Whitcomb 2007, Spier et al. 2012). Pesquisas mostram que insetos funcionam como vetores mecânicos na transmissão da bactéria, e estudos recentes demonstram que Haematobia irritans, Musca domestica e Stomoxys calcitrans são vetores paratênicos de $C p$ bv equi (Yeruham et al. 1996, Spier 2008).

A transmissão da bactéria ocorre pelo contato direto ou indireto, e o período de incubação varia de 7 a 28 dias (Doherr et al. 1998). A incidência depende das características do sistema imunológico dos equinos e de fatores ambientais supracitados.

A LU pode ser observada em cavalos de qualquer idade, embora haja baixa incidência em potros com menos de seis meses, devido à ingestão das imunoglobulinas maternas colostrais. Cavalos de menos de cinco anos de idade e os que são colocados para pastar no verão tem um maior risco de contrair a infeccção (Spier \& Whitcomb 2007). Já equinos mais velhos parecem ser menos susceptíveis, porque provavelmente já entraram em contato com essa bactéria ou com outras do mesmo gênero desenvolvendo imunidade cruzada (Doherr et al. 1998). A infecção por $C p$ bv equi não demonstra preferência por sexo ou raça (Pratt et al. 2005).

Dentre os fatores de risco associados com maior frequência ao desenvolvimento da infecção por Corynebacterium pseudotuberculosis em equinos estão: higiene deficiente dos estábulos (Zavoshti et al. 2009), idade, insetos em quantidade significativa, cavalos em ambientes abertos, estábulos superpovoados e atividade ao ar livre durante as épocas secas (Doherr et al. 1998). Smith \& Robinson (1981), durante um surto de $C p$ bv equi em potros, recuperaram a bactéria de teias de aranha nas baias que os potros doentes habitavam, e concluíram que aerossóis e poeira são fatores de risco. Recentemente, foi comprovada a transmissão mecânica da bactéria para equinos por moscas domésticas (Barba et al. 2015).

\section{SINTOMATOLOGIA E FORMAS DE APRESENTAÇÃO CLÍNICA DA DOENÇA EM EQUIDEOS}

Corynebacterium pseudotuberculosis é capaz de causar duas formas de apresentação de doença clínica em equinos (Biberstein et al. 1971): a primeira caracteriza-se pela presença de abscessos externos e é a forma mais branda da infecção (Aleman et al. 1996); a segunda é conhecida como LU, e é a forma mais grave da doença, por ser uma manifestação sistêmica (Pratt et al. 2005). Em 2007, Spier \& Whitcomb atualizam essa descrição e relatam três formas da infecção por $C p$ bv equi: linfangite ulcerativa propriamente dita, também conhecida por doença dos membros; a segunda forma caracterizada por abscessos externos e a terceira representada pelo desenvolvimento de abscessos internos. A taxa de mortalidade entre aqueles que não recebem tratamento é de 100\% (Aleman et al. 1996, e já foi visto que o diagnóstico precoce e o tratamento correto de equinos são fatores de grande importância para um bom prognóstico da infecção interna em equinos por $C$. pseudotuberculosis, principalmente por análises de ultrassonografia, as quais direcionam para a obtenção de amostras para diagnóstico 
(Pratt et al. 2005). Em um estudo mais recente, nenhuma correlação foi encontrada entre a cepa de C. pseudotuberculosis envolvida na infecção e o tipo de apresentação clínica, localização e extensão das lesões; os autores propõem que as diferenças fenotípicas encontradas durante as culturas in vitro não são relacionadas com a apresentação de sintomas clínicos da doença (Britz et al. 2014). Também títulos de anticorpos específicos, obtidos por ensaio de inibição da hemólise sinérgica, não são marcadores para a diferenciação da forma interna com a apresentação externa da doença (Jeske et al. 2013).

Na forma mais grave da doença, também chamada de peito ou febre de pombo, os sinais clínicos são comuns aos de várias outras infecções como anorexia, claudicação, hipertermia, letargia e perda de peso. Entretanto, edema na região do abscesso, feridas que não cicatrizam e dermatite ventral, conjugados com os sinais supracitados, são fortes indicadores de corinebacteriose equina (Hall et al. 2001). Os abscessos podem se desenvolver em um ou vários locais, assim como o $\mathrm{Cp}$ bv ovis em bovinos, ovinos e caprinos (Motta et al. 2010). As localizações mais comuns de aparecimento dos abcessos são as regiões peitoral, axilar, parte ventral do abdômen e região inguinal (Aleman et al. 1996), prepúcio, escroto, glândulas mamárias, membros e cabeça; entre as menos comuns, mas já relatadas, estão as regiões do tórax, pescoço, glândulas parótidas, bolsa gutural, laringe, flancos, umbigo, cauda e reto (Hall et al. 2001).

A forma sistêmica pode se manifestar como pericardite e pleurite, com derrame séptico (Perkins et al. 2004), aborto em éguas aparentemente saudáveis (Poonacha \& Donahue 1995), osteomielite e artrite séptica (Nogradi et al. 2012), meningite e meningoencefalomielite (Toth et al. 2012) e enterite (Woolcock et al. 1979). Os órgãos mais envolvidos são o fígado, pulmões, útero, rins e o baço (Falcon et al. 1985, Poonacha \& Donahue 1995, Lazzari et al. 1997, Hall et al. 2001, Spier \& Whitcomb 2007, Motta et al. 2010). São manifestações frequentemente encontradas na forma sistêmica: hepatomegalia, fibrose hepática, hepatite crônica, pleuropneumonia, doença renal crônica com fibrose, nefrite crônica e pielonefrite, abscessos esplênicos, cólica prolongada e intermitente, alteração no trato respiratório, dor abdominal, abscessos internos e externos concorrentes ou não, edema ventral, diarreia, ulceração bucal, petéquias nas membranas mucosas, púrpura hemorrágica, taquicardia e taquipnéia (Rumbaugh et al. 1978, Pratt et al. 2005).

Segundo Pratt et al. (2005), a LU é uma doença difusa dos membros. Quando a doença se cronifica, os membros tornam-se edemaciados, ocorrendo celulite e paniculite (Farstvedt et al. 2004), e consequentemente o animal desenvolve sinaiscomo claudicação grave, hipertermia, letargia, anorexia, fraqueza e perda de peso. Sinais de doença sistêmica podem estar presentes, pois o mesmo animal pode apresentar uma, duas ou as três formas da doença ao mesmo tempo (Spier \& Whitcomb 2007). 0 início da LU é lento e vai se cronificando, com a formação de abscessos ao longo do vaso linfático afetado, que se encontra duro e firme. Os abscessos exsudam pus espesso, inodoro, esverdeado e com rajas de sangue (Quinn et al. 2005).

\section{DIAGNÓSTICO DA INFECÇÃO EM EQUINOS}

No Brasil, especificamente, não há relatos de casos de infecção de cavalos por Corynebacterium pseudotuberculosis, e a ausência de diagnóstico dessa infecção provavelmente se deve ao fato de que a manifestação clínica da corinebacteriose em equinos é muito similar à de outras infecções (Motta et al. 2010). 0 diagnóstico diferencial deve ser feito em caso de suspeitas de mormo, dermatofilose, celulite estafilocócica, infecções por Streptococcus, Actinomycetes, Corynebacterium pyogenes e Rhodococcus equi (Yeruham et al. 1996, Motta et al. 2010).

Atualmente o diagnóstico dessa doença é realizado utilizando ferramentas simples, tais como a análise dos sinais clínicos, achados clínico-patológicos, sorologia para pesquisa de anticorpos e diagnóstico por imagem (Spier \& Whitcomb 2007); infelizmente, o exame sorológico, através de ELISA, não está comercialmente disponível no Brasil, sendo realizado apenas para estudos científicos, em algumas instituições acadêmicas, mas ainda direcionado para pequenos ruminantes (Rebouças et al. 2013). Tem-se também como opção de ensaio imunológico, para caprinos e ovinos, a dosagem de interferon-gama produzido após o estímulo específico de leucócitos do sangue periférico com antígenos de $C$. pseudotuberculosis, mas também não disponível comercialmente (Rebouças et al. 2011).

0 diagnóstico definitivo deve ocorrer através do isolamento e identificação do agente, com obtenção de amostras e cultivo em laboratório, associados a provas bioquímicas (Spier \& Whitcomb 2007) e técnicas de biologia molecular (Quinn et al. 2005, Soares et al. 2013b). A partir de biópsia de material purulento dos linfonodos e consequente cultura, foi desenvolvido ensaio de reação em cadeia da polimerase (PCR) do tipo multiplex, no qual, através da amplificação de três fragmentos específicos do genoma da bactéria, foi possível realizar uma detecção mais sensível e específica de C. pseudotuberculosis, com uma diferenciação mais acurada de outras bactérias filogeneticamente próximas, tais como Corynebacterium ulcerans, Corynebacterium diphteriae, Corynebacterium renale e Arcanobacterium pyogenes (Pacheco et al. 2007).

A diferenciação de $C p$ bv equi em relação a $C p$ bv ovis pode ser feita através de provas bioquímicas, como a capacidade de redução de nitrato. Entretanto, há possibilidade dessa bactéria se apresentar sob a forma de nitrato-negativo (Abreu et al. 2008a). A técnica de ERIC-PCR (reação em cadeia da polimerase de regiões consenso intergênicas repetitivas de enterobactérias), tornou possível diferenciar $C p$ bv equi de $C p$ bv ovis, sendo esta técnica atualmente descrita como sendo a mais acurada para realizar esse tipo de genotipagem em C. pseudotuberculosis (Dorneles et al. 2014).

As análises do soro e plasma são armas acessórias no diagnóstico clínico patológico. A anemia que se desenvolve durante a infecção em equinos com $C p$ bv equi propicia o aumento da concentração de fibrinogênio e da proteína total plasmáticos, com consequente hipergamaglobulinemia. Outros achados muito comuns nos animais que desenvolveram tanto a forma interna quanto a externa da doença foram leucocitose associada com neutrofilia e trombocito- 
penia (Pratt et al. 2005). Mas deve-se citar que essas alterações não são específicas para infecções com $C$. pseudotuberculosis, e podem ser observadas em outras doenças bacterianas de equideos.

Como diagnóstico diferencial, deve-se citar mais especificamente algumas doenças bacterianas de equídeos, tais como o mormo ou lamparão, causada pela bactéria Burkholderia mallei, que acomete principalmente os equinos, mas pode infectar também os muares e asininos, e considerada uma doença emergente desses animais no Brasil, principalmente na região nordeste do Brasil (Mota et al. 2000 ), na qual onde se encontram os rebanhos de pequenos ruminantes com as maiores prevalências de linfadenite caseosa no país. Também é necessária a diferenciação entre a LU causada por C. pseudotuberculosis e a dermatofilose, a qual tem como agente etiológico a bactéria Gram-positiva Dermatophilus congolensis; essa doença pode ter como manifestações clínicas febre, desidratação, congestão de mucosas, edema ventral e linfangite bilateral nos membros pélvicos (Melo et al. 2009). Dessa forma, é extremamente importante que ocorra a associação de uma completa anamnese clínica com ensaios sorológicos específicos e sensíveis, aliados ao isolamento do agente etiológico, suportado por provas bioquímicas e ensaios moleculares.

\section{TRATAMENTO}

O tratamento varia de acordo com a manifestação clínica da doença (Doherr et al. 1998). Quando o animal padece com a forma externa e superficial da doença, o tratamento recomendado é lancetar o abcesso, caso este não tenha se rompido naturalmente (Pratt et al. 2005). A drenagem e desinfecção com soluções à base de iodo a $10 \%$ desses pontos são frequentemente recomendadas; a retirada cirúrgica é indicada especialmente para os abscessos profundos musculares (Quinn et al. 2005, Spier \& Whitcomb 2007, Santiago et al. 2010). A antibioticoterapia deverá ser pensada nos casos de abscessos externos disseminados ou na manifestação clássica da LU, e deverá ser intensiva, especialmente se a formação de biofilme estiver instalada (Mah \& O'Toole 2001, Clutterbuck et al. 2007).

Injeções parenterais de penicilina, tetraciclina ou penicilina G procaína na concentração de 20.000 a 80.000 $\mathrm{UI} / \mathrm{kg}$ intramuscular por 30 dias ou mais é recomendada, especialmente se a fibrose estiver instalada (Radostits et al. 2007). Também é relatado o uso de penicilina potássica (20.000 a $40.000 \mathrm{UI} / \mathrm{kg}$ ), pura ou combinada com sulfonamidas a $15 \mathrm{mg} / \mathrm{kg}$ (Rose et al. 2000). Outras drogas citadas no tratamento de $C p$ bv ovis tem sido oxitetraciclina, eritromicina, sulfonamidas-trimetropina, rifampicina, florfenicol e ciprofloxacina (Prescott \& Yelding 1990, Senturk \& Temizel 2006, Pereira et al. 2010)

Já foi descrita a resistência de C. pseudotuberculosis a alguns antibióticos. Em um estudo com 54 isolados de $C$. pseudotuberculosis, foi detectada resistência para aminoglicosídeos, nitrofuranos e polimixinas (Judson \& Songer 1991). Em outro estudo, realizado no estado de Pernambuco, somente uma baixa porcentagem de isolados da bactéria teve sensibilidade a cefazolina, norfloxacina, amoxicilina ciprofloxacina, cloranfenicol e tetraciclina (Abreu et al. 2008b). Mais recentemente, foi visto em um estudo que 196 amostras de C. pseudotuberculosis isoladas de equinos não tiveram grande variação em seus perfis de sensibilidade a antibióticos (Rhodes et al. 2015). Mas deve-se levar em consideração também o fato de que, com a formação e desenvolvimento das lesões granulomatosas, o tratamento com antibióticos se torna um procedimento com baixa possibilidade de sucesso, pois a formação da cápsula do abscesso torna quase impossível a difusão dos antibióticos para a região da lesão em si, impedindo o contato da bactéria com o fármaco (Santiago et al. 2013).

\section{PREVENÇÃO}

Existem métodos simples, rápidos e baratos que os proprietários devem ser estimulados constantemente para prevenir a infecção com $C p$ bv equi, tais como: higiene dos estábulos, baias, pastos e picadeiros, controle constante de vetores, isolamento dos animais infectados, e evitar a aglomeração constante da população equina (Davies 2003, Zavoshti et al. 2009).

No controle do ambiente e de utensílios, alguns desinfetantes como hipoclorito de sódio a $2,5 \%$, cloro a $20 \%$, permanganato de potássio a $5 \%$ e peróxido de hidrogênio podem ser utilizados com boa eficiência (Xu et al. 1996, Santiago et al. 2010). Uma das principais metodologias de prevenção é evitar a contaminação do ambiente com material caseoso originário da abertura da lesão, seja espontânea ou por meio de procedimento cirúrgico. Com isso, além do isolamento do animal, recomenda-se intenso cuidado ao realizar a drenagem cirúrgica dos abscessos (Dorella et al. 2006).

Esforços têm sido direcionados para evitar a abertura da lesão caseosa, através da inoculação de agentes antissépticos dentro do abscesso, mas esse procedimento ainda apresenta diversos efeitos colaterais aos animais, como já descrito para pequenos ruminantes (Santiago et al. 2013). Também o acompanhamento sorológico frequente, através da detecção de anticorpos anti-C. pseudotuberculosis ou quantificação da produção específica de interferon-gama (Rebouças et al. 2011; Rebouças et al. 2013), como já descrito para caprinos e ovinos, pode ser um grande fator para evitar que mais animais dentro de um rebanho sejam infectados.

Não existe descrição na literatura sobre a imunoprofilaxia da infecção por C. pseudotuberculosis em equinos. Para caprinos e ovinos, existem vacinas comercialmente disponíveis, baseadas principalmente em cepas atenuadas da bactéria e na exotoxina fosfolipase $D$ recombinante inativada, mas essas vacinas agem diminuindo a intensidade das manifestações clínicas, e estudos moleculares e de bioinformática tem sido conduzidos no sentido de descobrir novos alvos para desenvolvimento de imunógenos mais eficientes (Hassan et al. 2014, Radusky et al. 2015). Esses estudos moleculares sofreram grande avanço após o sequenciamento do genoma de diversas cepas de C. pseudotuberculosis, e modelos vacinais baseados em bactérias recombinantes têm apresentado resultados promissores (Ribeiro et al. 2014, Silva et al. 2014). Uma promessa tem sido a análise proteômica de proteínas reconhecidas por 
soros de pequenos ruminantes naturalmente infectados pela bactéria, a qual tem revelado novos alvos para o desenvolvimento de vacinas (Seyffert et al. 2014). Apesar das pesquisas em vacinas estarem direcionadas, em sua grande parte, para pequenos ruminantes, as mesmas podem ser importantes para fundamentar novos antígenos a serem empregados na imunoprofilaxia da doença em equídeos.

\section{CONSIDERAÇÕES FINAIS}

A infecção por Corynebacterium pseudotuberculosis em equinos é encontrada mundialmente dispersa, podendo levar a diferentes sintomatologias clínicas, muitas das vezes não específicas e que podem ser confundidas com outras patologias.

No Brasil não há relatos científicos de isolamento da bactéria de equinos, mas como estão presentes diversos fatores de risco, presume-se que ocorra uma subnotificação da doença. É necessário investigar melhor os casos suspeitos da doença em equinos, através da realização do isolamento e identificação corretos do agente infeccioso, para poder realizar tratamento e prevenção mais eficazes e direcionados especificamente para C. pseudotuberculosis.

\section{REFERÊNCIAS}

Abreu S.R.O., Mota R.A., Forner O., Pinheiro Júnior J.W., Pereira R.R.B., Castro R.S., Elisei C., Soares C.S., Araújo F.R. \& Madureira R.C. 2008a. Comparação genotípica de isolados de Corynebacterium pseudotuberculosis de caprinos e ovinos do sertão de Pernambuco. Pesq. Vet. Bras. 28(10):481-487

Abreu S.R.O., Mota R.A., Pinheiro Júnior J.W., Rosinha G.M.S. \& Castro R.S. 2008b. Perfil de sensibilidade antimicrobiana in vitro de isolados de Corynebacterium pseudotuberculosis de caprinos e ovinos com linfadenite caseosa no sertão de Pernambuco, Brasil. Vet. Zootec. 15(3):502509.

Aleman M., Spier S.J., Wilson W.D. \& Doherr M. 1996. Corynebacterium pseudotuberulosis infection in horses: 538 cases (1982-1993). J. Am. Vet. Med. Assoc. 209(4):804-809.

Augustine J.L. \& Renshaw H.W. 1986. Survival of Corynebacterium pseudotuberculosis in axenic purulent exudate on common barnyard fomites. Am. J. Vet. Res. 47(4):713-715.

Baird G.J. \& Fontaine M.C. 2007. Corynebacterium pseudotuberculosis and its role in ovine caseous lymphadenitis. J. Comp. Pathol. 137(4):179210.

Barba M., Stewart A.J., Passler T., Wooldridge A.A., van Santen E., Chamorro M.F., Cattley R.C., Hathcock T., Hogsette J.A. \& Hu XP. 2015. Experimental transmission of Corynebacterium pseudotuberculosis biovar equi in horses by house flies. J. Vet. Intern. Med. 29(2):636-643.

Bauwens L., Van Dyck E., De Meurichy W. \& Piot P. 1987. Corynebacterium equi pneumonia in three Baikal seals (Pusa sibirica). Aq. Mam. 13:17-22.

Ben-Dov E., Ben Yosef D.Z., Pavlov V. \& Kushmaro A. 2009. Corynebacterium maris sp. nov., a marine bacterium isolated from the mucus of the coral Fungia granulosa. Int. J. Syst. Evol. Microbiol. 59(10):2458-2463.

Biberstein E.L., Knight H.D. \& Jang S. 1971. Two biotypes of Corynebacterium pseudotuberculosis. Vet. Rec. 89(26):691-692.

Braverman Y., Chizov-Ginzburg A., Saran A. \& Winkler M. 1999. The role of houseflies (Musca domestica) in harbouring Corynebacterium pseudotuberculosis in dairy herds in Israel. Rev. Sci. Technol. 18(3):681690.

Brennan N.M., Brown R., Goodfellow M., Ward A.C., Beresford T.P., Simpson P.J., Fox P.F. \& Cogan T.M. 2001. Corynebacterium mooreparkense sp. nov. isolated from the surface of a smear-ripened cheese. Int. J. Syst. Evol. Microbiol. 51(3):843-852
Britz E., Spier S.J., Kass P.H., Edman J.M. \& Foley J.E. 2014. The relationship between Corynebacterium pseudotuberculosis biovar equi phenotype with location and extent of lesions in horses. Vet. J. 200(2):282-6.

Carne H.R. \& Onon E.O. 1978. Action of Corynebacterium ovis exotoxin on endothelial cells of blood vessels. Nature 271:246-248.

Chen H.H., Li W.J., Tang S.K., Kroppenstedt R.M., Stackebrandt E., Xu L.H. \& Jiang C.L. 2004. Corynebacterium halotolerans sp. nov., isolated from saline soil in the west of China. Int. J. Syst. Evol. Microbiol. 54(3):779-782.

Clutterbuck A.L., Woods E.J., Knottenbelt D.C., Clegg P.D., Cochrane C.A. \& Percival S.L. 2007. Biofilms and their relevance to veterinary medicine. Vet. Microbiol. 121(1/2):1-17.

Collins M.D., Hoyles L., Foster G. \& Falsen E. 2004. Corynebacterium caspium sp. nov., from a Caspian seal (Phoca caspica). Int. J. Syst. Evol. Microbiol. 54(3):925-928.

Costa L.R., Spier S.J. \& Hirsh D.C. 1998. Comparative molecular characterization of Corynebacterium pseudotuberculosis of different origin. Vet. Microbiol. 62(2):135-43.

Cuevas W.A. \& Songer J.G. 1993. Arcanobacterium haemolyticum phospholipase D is genetically and functionally similar to Corynebacterium pseudotuberculosis phospholipase D. Infect. Immun. 61(10):4310-4316.

D’Afonseca V., Moraes P.M., Dorella F.A., Pacheco L.G., Meyer R., Portela R.W., Miyoshi A. \& Azevedo V. 2008. A description of genes of Corynebacterium pseudotuberculosis useful in diagnostics and vaccine applications. Genet. Mol. Res. 7(1):252-260.

Davies D. 2003. Understanding biofilm resistance to antibacterial agents. Nat. Rev. Drug Discov. 2:114-122.

Doherr M.G., Carpenter T.E., Hanson K.M., Wilson W.D. \& Gardner I.A. 1998. Risk factors associated with Corynebacterium pseudotuberculosis infection in California horses. Prev. Vet. Med. 35:229-239.

Dorella F.A., Pacheco L.G., Oliveira S.C., Miyoshi A. \& Azevedo V. 2006. Corynebacterium pseudotuberculosis: microbiology, biochemical properties, pathogenesis and molecular studies of virulence. Vet. Res. 37(2):201-18

Dorneles E.M., Santana J.A., Ribeiro D., Dorella F.A., Guimarães A.S., Moawad M.S., Selim S.A., Garaldi A.L., Miyoshi A., Ribeiro M.G., Gouveia A.M., Azevedo V., Heinemann M.B. \& Lage A.P. 2014. Evaluation of ERIC-PCR as genotyping method for Corynebacterium pseudotuberculosis isolates. PLoS One 9(6):e98758.

Du Z.J., Jordan E.M., Rooney A.P., Chen G.J. \& Austin B. 2010. Corynebacterium marinum sp. nov. isolated from coastal sediment. Int. J. Syst. Evol. Microbiol. 60(8):1944-1947.

Falcon J., Smith B.P., O'Brien T.R., Carlson G.P. \& Biberstein E. 1985. Clinical and radiographic findings in Corynebacterium equi pneumonia of foals. J. Am. Vet. Med. Assoc. 186(6):593-599.

Farstvedt E.G., Hendrickson D.A., Dickenson C.E. \& Spier S.J. 2004. Treatment of suppurative facial cellulitis and panniculitis caused by Corynebacterium pseudotuberculosis in two horses. J. Am. Vet. Med. 224(7): 1139-1142.

Hall-Stoodley L., Costerton J.W. \& Stoodley P. 2004. Bacterial biofilms: from the natural environment to infectious diseases. Nat. Rev. Microbiol. 2(2):95-108.

Hall K., McCluskey B.J. \& Cunningham W. 2001. Corynebacterium pseudotuberculosis infections (Pigeon Fever) in horses in Western Colorado: an epidemiological investigation. J. Eq. Vet. Sci. 21(6):284-286.

Hall I.C. \& Fisher C.W. 1915. Supurative lesions in horses and a calf of California due to the diphteroid bacillus of Peïsz-Nocard. J. Am. Vet. Med. Assoc. 1:18-30.

Hassan S.S., Tiwari S., Guimarães L.C., Jamal S.B., Folador E., Sharma N.B., de Castro Soares S., Almeida S., Ali A., Islam A., Póvoa F.D., de Abreu V.A., Jain N., Bhattacharya A., Juneja L., Miyoshi A., Silva A., Barh D., Turjanski A., Azevedo V. \& Ferreira RS. 2014. Proteome scale comparative modeling for conserved drug and vaccine targets identification in Corynebacterium pseudotuberculosis. BMC Genomics 15(7):S3

Hodgson A.L., Krywult J., Corner L.A., Rothel J.S. \& Radford A.J. 1992. Rational attenuation of Corynebacterium pseudotuberculosis: potential cheesy gland vaccine and live delivery vehicle. Infect. Immun. 60(7):2900-2905. 
Jeske J.M., Spier S.J., Whitcomb M.B., Pusterla N. \& Gardner I.A. 2013. Use of antibody titers measured via serum synergistic hemolysis inhibition testing to predict internal Corynebacterium pseudotuberculosis infection in horses. J. Am. Vet. Med. Assoc. 242(1):86-92.

Judson R. \& Songer J.G. 1991. Corynebacterium pseudotuberculosis: in vitro susceptibility to 39 antimicrobial agents.Vet. Microbiol. 27(2):145-150.

Khamis A., Raoult D. \& La Scola B. 2004. rpoB gene sequencing for identification of Corynebacterium species. J. Clin. Microbiol. 42(9):3925-31.

Lazzari A., De Vargas A.C., Dutra V., Costa M.M. \& Flores L.A.S. 1997. Aspectos epidemiológicos do Rhodococcus equi em eqüinos do município de Bagé, RS, Brasil. Ciência Rural 27:3.

Mah T.F. \& O’Toole G.A. 2001. Mechanisms of biofilm resistance to antimicrobial agents. Trends Microbiol. 9(1):34-39.

McNamara P.J., Cuevas W.A. \& Songer J.G. 1995. Toxic phospholipases D of Corynebacterium pseudotuberculosis, C. ulcerans and Arcanobacterium haemolyticum: cloning and sequence homology. Gene 156(1):113-118.

McNamara P.J., Bradley G.A. \& Songer J.G. 1994. Targeted mutagenesis of the phospholipase D gene results in decreased virulence of Corynebacterium pseudotuberculosis. Mol. Microbiol. 12(6):921-930.

Melo U.P., Ferreira C., Fiório R.C., Araújo T.B.S. \& Santos P.M.P. 2009. Linfangite aguda e síndrome da resposta inflamatória sistêmica secundárias à dermatofilose generalizada em um equino. Acta Vet. Brasil. 3(1):33-37.

Mota R.A., Brito M.F., Castro F.J.C. \& Massa M. 2000. Mormo em equídeos nos estados de Pernambuco e Alagoas. Pesq. Vet. Bras. 20(4):155-159.

Motta R.G., Cremasco A.C.M. \& Ribeiro M.G. 2010. Infecções por Corynebacterium pseudotuberculosis em animais de produção. Vet. Zootec. 17(2):200-213.

Moura-Costa L.F. 2002. Corynebacterium pseudotuberculosis, the ethiological agent of the caseous lymphadenitis in goats. Revta Ciênc. Méd. Biol. 1(1):105-115.

Moura Costa M.D. 1973. Linfadenite caseosa dos caprinos no Estado da Bahia. Distribuição geográfica da doença. Bibl. Inst. Biol. Bahia 12(1):1-7.

Nogradi N., Spier S.J., Toth B. \& Vaughan B. 2012. Musculoskeletal Corynebacterium pseudotuberculosis infection in horses: 35 cases (1999-2009). J. Am. Vet. Med. Assoc. 241(6):771-777.

Olson M.E., Ceri H., Morck D.W., Buret A.G. \& Read R.R. 2002. Biofilm bacteria: formation and comparative susceptibility to antibiotics. Can. J. Vet. Res. 66(2):86-92.

O'Toole G., Kaplan H.B. \& Kolter R. 2000. Biofilm formation as microbial development. Annu. Rev. Microbiol. 54:49-79.

Pacheco L.G., Pena R.R., Castro T.L., Dorella F.A., Bahia R.C., Carminati R., Frota M.N., Oliveira S.C., Meyer R., Alves F.S., Miyoshi A. \& Azevedo V. 2007. Multiplex PCR assay for identification of Corynebacterium pseudotuberculosis from pure cultures and for rapid detection of this pathogen in clinical samples. J. Med. Microbiol. 56(4):480-486.

Pereira W.A.B., Nogueira G.M., Paro P.H.Z., Espinoza M.F. \& Leite A.D. 2010. Linfangite ulcerativa em um touro nelore: relato de caso. Revta Cient. Eletr. Med. Vet. 14:1-5.

Perkins S.L., Magdesian K.G., Thomas W.P. \& Spier S.J. 2004. Pericarditis and pleuritis caused by Corynebacterium pseudotuberculosis in a horse. J. Am. Vet. Med. Assoc. 224(7):1133-1138.

Pethick F.E., Lainson A.F., Yaga R., Flockhart A., Smith D.G., Donachie W., Cerdeira L.T., Silva A., Bol E., Lopes T.S., Barbosa M.S., Pinto A.C., Dos Santos A.R., Soares S.C., Almeida S.S., Guimarães L.C., Aburjaile F.F., Abreu V.A., Ribeiro D., Fiaux K.K., Diniz C.A., Barbosa E.G., Pereira U.P., Hassan S.S., Ali A., Bakhtiar S.M., Dorella F.A., Carneiro A.R., Ramos R.T., Rocha F.S., Schneider M.P., Miyoshi A., Azevedo V. \& Fontaine M.C. 2012. Complete genome sequence of Corynebacterium pseudotuberculosis strain 1/06-A, isolated from a horse in North America. J. Bacteriol. 194(16):4476.

Poonacha K.B. \& Donahue J.M. 1995. Abortion in a mare associated with Coryrtebacterium pseudotuberculosis infection. J. Vet. Diagn. Invest. 7:563-564.

Pratt S.M., Spier S.J., Carroll S.P., Vaughan B., Whitcomb M.B. \& Wilson W.D. 2005. Evaluation of clinical characteristics, diagnostic test results, and outcome in horses with internal infection caused by Corynebacte- rium pseudotuberculosis: 30 cases (1995-2003). J. Am. Vet. Med. Assoc. 227(3):441-448.

Prescott J. \& Yelding K.H. 1990. In vitro susceptibility of selected veterinary bacterial pathogens to ciprofloxacin, enrofloxacin and norfloxacin. Can. J. Vet. Res. 54:195-197.

Proft T. \& Baker E.N. 2009. Pili in Gram-negative and Gram-positive bacteria - structure, assembly and their role in disease. Cell Mol. Life Sci. 66(4):613-635.

Pugh G.D. 2004. Sheep and Goat Medicine. Elsevier, New York.

Quinn J.P. 2005. Microbiologia Veterinária e Doenças infecciosas. Artmed, Porto Alegre, p.67-70.

Radostits O.M. 2007. Veterinary Medicine: a textbook of the diseases of cattle, sheep, pigs, goats and horses. Bailliere Tindall, Philadelphia, p.830-839.

Radusky L.G., Hassan S., Lanzarotti E., Tiwari S., Jamal S., Ali J., Ali A., Ferreira R., Barh D., Silva A., Turjanski A.G. \& Azevedo V.A. 2015. An integrated structural proteomics approach along the druggable genome of Corynebacterium pseudotuberculosis species for putative druggable targets. BMC Genomics. 16(5):S9.

Rebouças M.F., Portela R.W., Lima D.D., Loureiro D., Bastos B.L., Moura-Costa L.F., Vale V.L., Miyoshi A., Azevedo V. \& Meyer R. 2011. Corynebacterium pseudotuberculosis secreted antigen-induced specific gamma-interferon production by peripheral blood leukocytes: potential diagnostic marker for caseous lymphadenitis in sheep and goats. J. Vet. Diagn. Invest. 2011 23(2):213-220.

Rebouças M.F., Loureiro D., Bastos B.L., Moura-Costa L.F., Hanna S.A., Azevedo V., Meyer R. \& Portela R.W. 2013. Desenvolvimento de ELISA indireto para detectar anticorpos específicos contra Corynebacterium pseudotuberculosis em ovinos utilizando o supernadante de cultura da cepa T1 como antígeno. Pesq. Vet. Bras. 33(11):1296-1302.

Rhodes D.M., Magdesian K.G., Byrne B.A., Kass P.H., Edman J. \& Spier S.J. 2015. Minimum inhibitory concentrations of equine Corynebacterium pseudotuberculosis isolates (1996-2012). J. Vet. Intern. Med. 29(1):327332.

Ribeiro D., Rocha F.S., Leite K.M., Soares S.C., Silva A., Portela R.W., Meyer R., Miyoshi A., Oliveira S.C., Azevedo V. \& Dorella F.A. 2014. An iron-acquisition-deficient mutant of Corynebacterium pseudotuberculosis efficiently protects mice against challenge. Vet. Res. 45(1):28.

Ribeiro M.G., Belotta A.F., Fernandes M.C., Guena R., Nardi Júnior G., Lara G.H.B., Giuffrida R. \& Zamprogna T.O. 2011. Citologia aspirativa no diagnóstico da linfadenite em ovinos. Pesq. Vet. Bras. 31(10):839-843.

Robinson R.C. 1982 Epidemiological and bacteriological studies of Corynebacterium equi isolates from Californian farms. J. Reprod. Fertil. 32:477480.

Rose R. 2000. Manual of Equine Practice. W.B. Saunders, Philadelphia, p.497-498.

Rumbaugh G.E., Smith B.P. \& Carlson G.P. 1978. Internal abdominal abscesses in the horse: a study of 25 cases. J. Am. Vet. Med. Assoc. 172(3): 304-309.

Sá M.C.A., Veschi J.L.A., Santos G.B., Amanso E.A., Oliveira S.A.S., Mota R.A., Veneroni-Gouveia G. \& Costa M.M. 2013. Activity of disinfectants and biofilm production of Corynebacterium pseudotuberculosis. Pesq. Vet. Bras. 33(11):1319-1324.

Santiago L.B., Alves F.S.F., Pinheiro R.R., Santos V.W.S., Rodrigues A.S., Chapaval L., Brito I.F. \& Sousa F.G.C. 2010. Avaliação in vitro da sensibilidade da Corynebacterium pseudotuberculosis frente a diferentes tipos de antissépticos e desinfetantes e determinação de sua curva de crescimento. Arqs Inst. Biológico, São Paulo, 77(4):593-600.

Santiago L.B., Pinheiro R.R., Aves F.S.F., Santos V.W.S., Rodrigues A.S., Lima A.M.C., Oliveira E.L. \& Albuquerque F.H.M.A.R. 2013. In vivo evaluation of antiseptics and disinfectants on control of Caseous Lymphadenitis: clinical, haematological, serological and microbiological monitoring. Arq. Inst. Biol. 80(3):273-280.

Schumann W. 2007. Thermosensors in eubacteria: role and evolution. J. Biosci. 32(3):549-557. 
Senturk S. \& Temizel M. 2006. Clinical efficacy of rifamycin SV combined with oxytetracyclime in the treatment of caseous lymphadenitis in sheep. Vet. Rec. 159(7):216-217.

Seyffert N., Silva R.F., Jardin J., Silva W.M., Castro T.L., Tartaglia N.R., Santana K.T., Portela R.W., Silva A., Miyoshi A., Le Loir Y. \& Azevedo V. 2014. Serological proteome analysis of Corynebacterium pseudotuberculosis isolated from different hosts reveals novel candidates for prophylactics to control caseous lymphadenitis. Vet. Microbiol. 174(1-2):255-260.

Silva A., Schneider M.P., Cerdeira L., Barbosa M.S., Ramos R.T., Carneiro A.R., Santos R., Lima M., D’Afonseca V., Almeida S.S., Santos A.R., Soares S.C., Pinto A.C., Ali A., Dorella F.A., Rocha F., Abreu V.A., Trost E., Tauch A., Shpigel N., Miyoshi A. \& Azevedo V. 2011. Complete Genome Sequence of Corynebacterium pseudotuberculosis I19, a strain isolated from a cow in Israel with bovine mastitis. J. Bacteriol. 193(1)323-324.

Silva J.W., Droppa-Almeida D., Borsuk S., Azevedo V., Portela R.W., Miyoshi A., Rocha F.S., Dorella F.A., Vivas W.L., Padilha F.F., Hernández-Macedo M.L \& Lima-Verde I.B. 2014. Corynebacterium pseudotuberculosis cp09 mutant and cp40 recombinant protein partially protect mice against caseous lymphadenitis. BMC Vet. Res. 20(10):965.

Smith B.P. \& Robinson R.C. 1981. Studies of an outbreak of Corynebacterium equi pneumonia in foals. Eq. Vet. J. 13(4):223-228.

Soares S.C., Silva A., Trost E., Blom J., Ramos R., Carneiro A., Ali A., Santos A.R., Pinto A.C., Diniz C., Barbosa E.G., Dorella F.A., Aburjaile F., Rocha F.S., Nascimento K.K., Guimarães L.C., Almeida S., Hassan S.S., Bakhtiar S.M., Pereira U.P., Abreu V.A., Schneider M.P., Miyoshi A., Tauch A. \& Azevedo V. 2013. The Pan-Genome of the animal pathogen Corynebacterium pseudotuberculosis reveals differences in genome plasticity between the biovar ovis and equi strains. PLoS One 8(1):e53818.

Soares S.C., Trost E., Ramos R.T., Carneiro A.R., Santos A.R., Pinto A.C., Barbosa E., Aburjaile F., Ali A., Diniz C.A., Hassan S.S., Fiaux K., Guimarães L.C., Bakhtiar S.M., Pereira U., Almeida S.S., Abreu V.A., Rocha F.S., Dorella F.A., Miyoshi A., Silva A., Azevedo V. \& Tauch A. 2013. Genome sequence of Corynebacterium pseudotuberculosis biovar equi strain 258 and prediction of antigenic targets to improve biotechnological vaccine production. J. Biotechnol. 167(2):135-141

Songer J.G., Libby S.J., Iandolo J.J. \& Cuevas W.A. 1990. Cloning and expression of the phospholipase D gene of Corynebacterium pseudotuberculosis in Escherichia coli. Infect. Immun. 58(1):131-136.

Spier S. 2008. Corynebacterium pseudotuberculosis infection in horses: an emerging disease associated with climate change. Eq. Vet. Educ. 20:37-39.
Spier S.J. \& Whitcomb M.B. 2007. Equine Infectious Diseases. Elsevier Saunders, St Louis, p.263-269.

Spier S.J., Leutenegger C.M., Carroll S.P., Loye J.E., Pusterla J.B., Carpenter T.E., Mihalyi J.E. \& Madigan J.E. 2004. Use of a real-time polymerase chain reaction-based fluorogenic 5' nuclease assay to evaluate insect vectors of Corynebacterium pseudotuberculosis infections in horses. Am. J. Vet. Res. 65(6):829-834.

Spier S.J., Toth B., Edman J., Quave A., Habasha F., Garrick M. \& Byrne B.A. 2012. Survival of Corynebacterium pseudotuberculosis biovar equi in soil. Vet. Rec. 170(7):180.

Sutherland S.S., Hart R.A. \& Buller N.B. 1996. Genetic differences between nitrate-negative and nitratepositive $C$. pseudotuberculosis strains using restriction fragment length polymorphisms. Vet. Microbiol. 49(1/2):1-9.

Toth B., Aleman M., Nogradi N. \& Madigan J.E. 2012. Meningitis and meningoencephalomyelitis in horses: 28 cases (1985-2010). J. Am. Vet. Med. Assoc. 240(5):580-587.

Williamson L.H. 2001. Caseous lymphadenitis in small ruminants. Vet. Clin. North. Am., Food Anim. Pract. 17(2):359-371.

Woolcock J.B., Farmer A.M.T. \& Mutimer M.D. 1979. Selective Medium for Corynebacterium equi isolation. J. Clin. Microb. 9(5):640-642.

Wu C.Y., Zhuang L., Zhou S.G., Li F.B. \& He J. 2011. Corynebacterium humireducens sp. nov, an alkaliphilic, humic acid-reducing bacterium isolated from a microbial fuel cell. Int. J. Syst. Evol. Microbiol. 61(4):882887.

Yeruham I., Braverman Y., Shpigel N.Y., Chizov-Ginzburg A., Saran A. \& Winkler M. 1996. Mastitis in dairy cattle caused by Corynebacterium pseudotuberculosis and the feasibility of transmission by houseflies. I. Vet. Quart. 18(3):87-89.

Xu X., Stewart P.S. \& Chen X. 1996. Transport limitation of chlorine disinfection of Pseudomonas aeruginosa entrapped in alginate beads. Biotechnol. Bioeng. 49(1):93-100.

Zavoshti F.R., Khojine A.B.S. \& Mahpeikar H.A. 2009. A case report of ulcerative lymphangitis: a mini review of causes and current therapies. Turk. J. Vet. Anim. Sci. 33(6):525-528.

Zhou Z., Yuan M., Tang R., Chen M., Lin M. \& Zhang W. 2012. Corynebacterium deserti sp. nov., isolated from desert sand. Int. J. Syst. Evol. Microbiol. 62(4):791-794. 